\title{
Computational criteria for establishing reflex from plasticity to hardwiring
}

\author{
David Tam \\ From Nineteenth Annual Computational Neuroscience Meeting: CNS*2010 \\ San Antonio, TX, USA. 24-30 July 2010
}

The role of synaptic plasticity in associative learning has been explored extensively computationally. Yet, the computational mechanism for hardwiring of the neural circuit has only been addressed recently using a systems approach of whole-animal behavior based on an evolutionary process for survival $[1,2]$. In this paper, we will explore the computational criteria needed to establish the hardwiring of reflex. The traditional focus of synaptic plasticity is often placed on addressing the conditions in which the learning process occurs so as to alter the synaptic strength rather than the conditions in which the learning process should stop. This paper addresses the question of the conditions under which learning should stop and hardwiring begins. Rather than allowing the system to "over-learn" indefinitely, it is often desirable to stop the malleable process, and begin hardwiring the circuitry so that the system can respond faster and automatically as a reflex.

The establishment of reflex plays an important role in animal behavior such that no learning is required in order to respond to a stimulus. Thus, the question of how such reflex stimulus-response function is established from prior experience or passed on from previous generation is the focus of this paper. We have derived a set of conditions in which synaptic plasticity would stop and when hardwiring should begin. The criteria are based on the asymptote of the learning process in errorminimization that reaches a stable state. When the error measure is used as the criteria for determining the stability of learning, it can also be used to determine whether the error has reached a minimum. This minimization criterion can then be used as the condition for hardwiring the circuitry rather than allowing plasticity to continue indefinitely. Although the system may have

\footnotetext{
Correspondence: dtam@unt.edu
}

Department of Biological Sciences, University of North Texas, Denton,

TX 76203, USA reached a local minimum rather than a global minimum, it can serve as a "best practice" solution for a given associative learning stimulus-response function. In fact, most biological reflexes are often best practice solution for a given stimulus-response function with a local minimum rather than a global minimum solution. Such hardwired circuitry can then be passed onto the next generation as the initial condition for further refinement. This would improve the solution search time since the knowledge from prior experience is embedded in the initial conditions of the neural circuitry passed on from the previous generation. The further refinement of the reflex by the next generation iteration can then be performed by "conditioned reflex" where the innate reflex can be modified by fine adjustment to the stimulusresponse function.

Published: 20 July 2010

\section{References}

1. Tam D: EMOTION-I Model: A Biologically-Based Theoretical Framework for Deriving Emotional Context of Sensation in Autonomous Control Systems. The Open Cybernetics \& Systemics Journal 2007, 1:28-46.

2. Tam D: EMOTION-II Model: A Theoretical Framework for Happy Emotion as a Self-Assessment Measure Indicating the Degree-of-Fit (Congruency) between the Expectancy in Subjective and Objective Realities in Autonomous Control Systems. The Open Cybernetics \& Systemics Journal 2007, 1:47-60.

doi:10.1186/1471-2202-11-S1-P110

Cite this article as: Tam: Computational criteria for establishing reflex from plasticity to hardwiring. BMC Neuroscience 2010 11(Suppl 1):P110. 